\title{
Chemical approach to fabrication of semicontinuous Au nanolayers for SERS applications
}

\author{
Bartosz Bartosewicz, Magdalena Gajda-Rączka, Bartłomiej J. Jankiewicz* \\ Institute of Optoelectronics, Military University of Technology, Kaliskiego 2, 00-908 Warsaw, Poland
}

Received May 12, 2013; accepted June 28, 2013; published June 30, 2013

\begin{abstract}
The fabrication of semicontinuous $\mathrm{Au}$ films on glass slides by using a chemical approach was carried out to test their surface enhanced Raman scattering (SERS) properties. Scanning electron microscopy (SEM) and UV-Vis-NIR spectroscopy were used for characterization of the morphology and optical properties of fabricated gold nanolayers. The SERS properties of Au films were tested using $p$ mercaptoaniline (pMA). A maximum estimated SERS enhancement factor greater than $10^{6}$ can be achieved for this compound. SERS enhancement factors are shown to be dependent on the Au nanolayers' morphology.
\end{abstract}

Over the recent years the surface enhanced Raman spectroscopy (SERS) has become an important sensing tool for the highly sensitive detection of chemical and biological agents [1]. The SERS effect, mainly attributed to the electromagnetic field enhancement caused by localized surface plasmon excitation in metallic nanostructures through the incident laser light, is strongly dependent on the morphology of metallic nanostructures [2]. Thus, it is not surprising that numerous studies have been dedicated to the design and fabrication of highly active SERS substrates based on nanostructured films and metallic nanoparticles [3-5]. Examples of SERS active structures include colloid aggregates [6], substrates fabricated by nanosphere lithography (NSL) [7], adaptive silver films (ASF) fabricated by electron beam evaporation [8-9] and $\mathrm{Au}$ and Ag nanoshells [10-11]. An interesting approach to fabrication of SERS substrates was reported by Natan et al. [12]. Chemical reduction of gold salts with a weak reducing agent on functionalized glass slide covered with gold colloids yielded semicontinuous $\mathrm{Au}$ films [12-13]. Structures with similar morphologies and properties are accessible, for example, by electron beam evaporation [8]. However, this method requires more advanced instrumentation and is rather expensive.

In this work, the fabrication of semicontinuous $\mathrm{Au}$ films on glass substrates was carried out by using a modification of the method developed by Natan et al. [12]. The fabricated $\mathrm{Au}$ films were tested to determine their suitability for SERS measurements.

\footnotetext{
*E-mail: bjankiewicz@wat.edu.pl
}

The semicontinuous $\mathrm{Au}$ films described in this work were prepared as shown in Fig. 1. The microscope glass slides were cleaned in base piranha $\left(\mathrm{NH}_{4} \mathrm{OH}: \mathrm{H}_{2} \mathrm{O}_{2} 3: 1\right)$ and functionalized with $0.3 \mathrm{mM}$ (3-aminopropyl) trimethoxysilane (APTMS, Sigma Aldrich) in toluene at $100^{\circ} \mathrm{C}$ for 1 hour [14]. The $\mathrm{NH}_{2}$-functionalized slides were rinsed with ethanol and water and placed in a solution of gold nanoparticles (AuNPs) prepared by using Turkevich method for 12 hours (sample A in Fig. 2) [15]. Single AuNPs covered glass slides were then placed on the bottom of a Petri dish $(d=51 \mathrm{~mm})$ filled with $20 \mathrm{ml}$ of $3 \mathrm{mM} \mathrm{NH} \mathrm{NH}_{2} \mathrm{OH}$ (Sigma Aldrich). The growth of $\mathrm{Au}$ layers with various morphologies was induced by addition to the reaction a mixture of different volumes of $0.5 \% \mathrm{HAuCl}_{4}$ (Sigma Aldrich) made from a stock solution and stirring it for $30 \mathrm{~min}$ at room temperature (samples B-F in Fig. 2). Fabricated Au covered slides were rinsed thoroughly with water to remove unreacted reagents and stored in water before use.

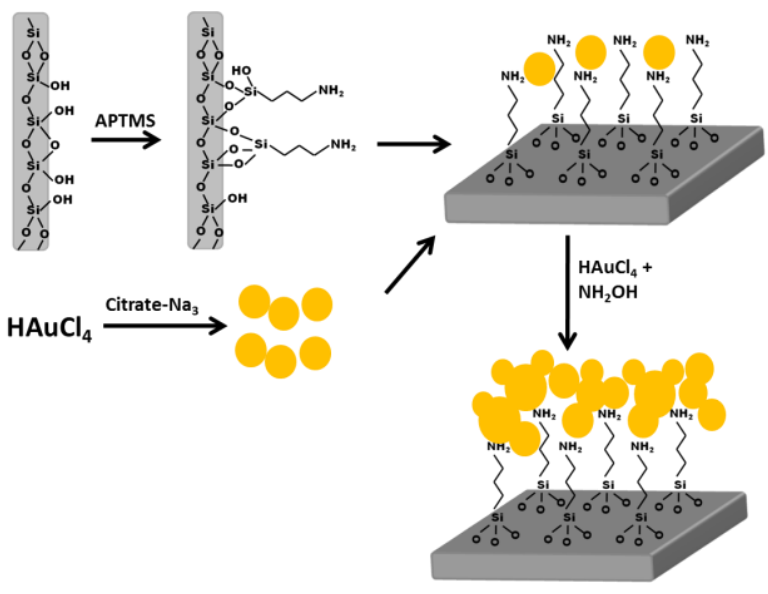

Fig. 1. Formation of semicontinuous Au nanolayer on glass slide.

The morphology and optical properties of the fabricated structures were characterized by using scanning electron microscopy (SEM) and UV-Vis-NIR spectrophotometry. The SEM images were obtained with a Quanta 3D FEG Dual Beam and UV-Vis-NIR spectra were measured using a Perkin-Elmer Lambda 900 spectrometer. 

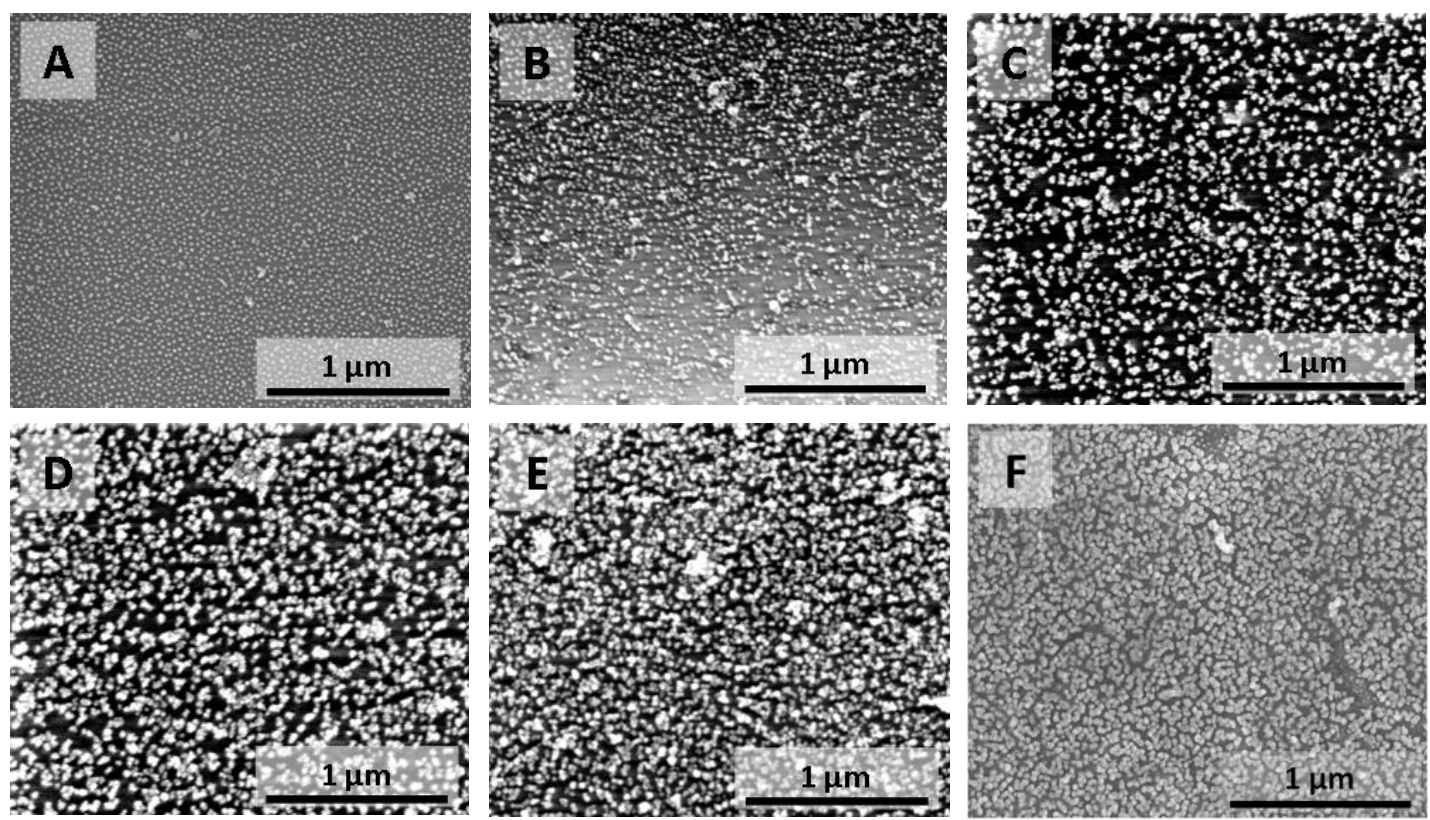

Fig. 2. SEM images of an AuNPs layer on amine functionalized glass (A) and Au nanolayers obtained by reduction of $30 \mu 1$ (B), $60 \mu 1$ (C), $90 \mu 1$ (D), $120 \mu \mathrm{l}(\mathrm{E})$ and $200 \mu \mathrm{l}(\mathrm{F})$ of $0.5 \%$ solution of $\mathrm{HAuCl}_{4}$ on AuNPs layer with a constant volume of $3 \mathrm{mM}$ solution of $\mathrm{NH}_{2} \mathrm{OH}$.

SERS measurements were carried out using Renishaw InVia Raman microscope with a laser beam $(785 \mathrm{~nm}$ excitation wavelength) directed to the sample through an $50 \times$ objective lens. $30 \mu \mathrm{l}$ of $50 \mu \mathrm{M} p$-mercaptoaniline (pMA) solution in ethanol was deposited on each sample so that it was uniformly evenly distributed over the sample surface, and the solvent was allowed to slowly evaporate.

The preparation of functionalized glass slides was performed by cleaning them with base piranha. In addition to removal of any impurities from the glass surface, the treatment with this strongly basic medium leads to the activation of surface silanol bonds before surface modification. The surface functionalization is an important step, which determines the number of AuNPs deposited on the surface as well as their arrangement. The glass surface may be functionalized using various organosilanes bearing functional groups with affinity to gold surfaces [16]. Based on our experience, among organosilanes with cyano $(-\mathrm{CN})$, thiol (-SH) and amino (- $\left.\mathrm{NH}_{2}\right)$ groups, the highest density of AuNPs on the surface was achieved for the latter ones, which were used in these studies. The treatment of clean and activated glass surfaces with APTMS yields $\mathrm{NH}_{2}$-functionalized slides. Depending on the organosilane used, as well as the exact method and the time of functionalization, the surface density of amino groups on the surface may vary from 1.5 to $8.9 / 100 \AA^{2}$. However, absolute densities with values higher than five indicate rough surface morphology [17].

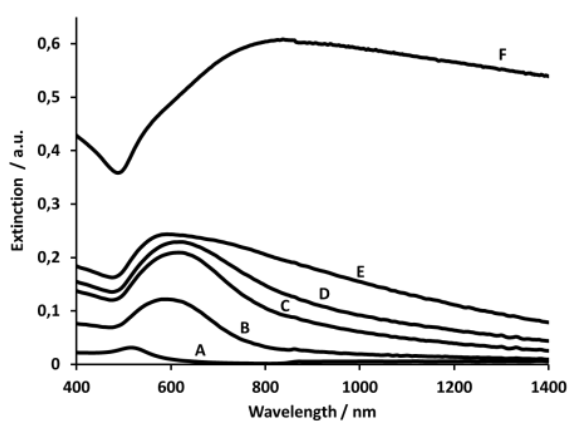

Fig. 3. UV-Vis-NIR spectra of AuNPs layer on amine functionalized glass (A) and Au nanolayers B-F shown in Fig. 2.

The deposition of 15-20nm AuNPs was carried out by placing amine glass slides in a solution containing AuNPs for 12 hours. This step yields monolayers of AuNPs (as shown in Fig. 2A) with high reproducibility if certain precautions are undertaken to prevent undesirable agglomeration of metal nanoparticles. For example, when growing Au layers (Fig. 1), the slides need to be attached to the bottom of Petri dishes with double-sided tape. This ensures that the slides remain still during stirring and also facilitates the deposition of AuNPs and growing Au layers only on one side of the slides. Further, in the last step of $\mathrm{Au}$ nanolayers preparation, AuNPs (Fig. 2A) covered glass slides should be placed in a solution of hydroxylamine and various volumes of $0.5 \%$ solution of $\mathrm{HAuCl}_{4}$ should be added while stirring the solution. After this, stirring should be continued for 30 minutes. This treatment ensures the formation of complex structures as a result 
of growth and coalescence of closely lying AuNPs. The changes to $\mathrm{Au}$ layers morphologies and optical properties with respect to added volume of $\mathrm{Au}$ salts are presented in Fig. 2 and in Fig. 3. It is important to mention that in the case of the discussed studies, the morphology of layers was controlled by changing the gold salts concentration, while in the studies reported by Natan et al., it was done by controlling substrate immersion time in shaken solutions of $\mathrm{HAuCl}_{4} / \mathrm{NH}_{2} \mathrm{OH}$ [12].

The characterization of the optical properties of fabricated substrates has shown that the deposition of AuNPs on functionalized slides yields structures whose optical properties closely resemble the optical properties of AuNPs solutions, with a characteristic plasmon resonance maximum near 520nm (Fig. 3A) [15]. Further reduction of gold salts on substrate (A) leads to a gradual increase of extinction, broadening of the peak and shift of $\lambda_{\max }$ to longer wavelengths, as shown in Fig. 3 (B-F). The change in optical properties can be explained, at least partially, by the shortening of interparticle spacings, which allows for electromagnetic interactions between $\mathrm{Au}$ nanostructures through a dipole coupling mechanism.

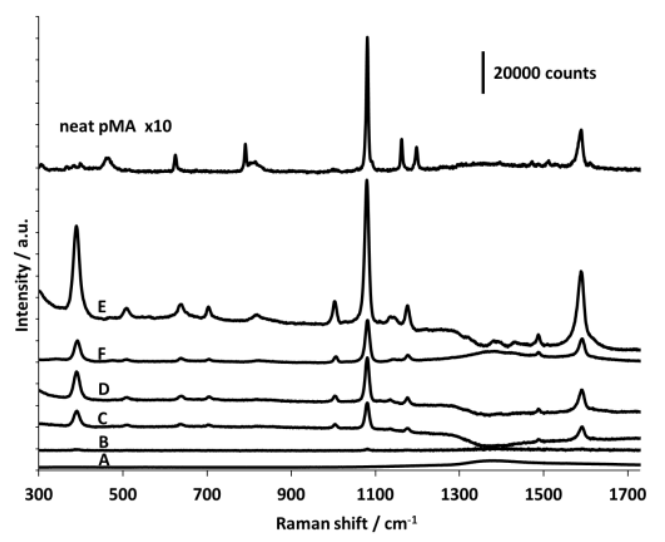

Fig. 4. SERS spectra of neat pMA and samples made by deposition of $30 \mu \mathrm{l}$ of $50 \mu \mathrm{M}$ pMA solution on layers of AuNPs on amine functionalized glass (A) and Au nanolayers (B-F) (shown in Fig. 2).

The SERS performance of the fabricated Au layers was evaluated using pMA (Fig. 4), the analyte commonly used for quantification of SERS enhancement factor (EF), due to its ability to form selfassembled monolayers (SAMs) with a known packing density on Au surfaces [18-19]. The SERS EFs were estimated using the following equation:

$$
E F=\frac{I_{S E R S} / N_{\text {surf }}}{I_{R S} / N_{v o l}}
$$

by comparing the ratios of the SERS peak intensity (I $\mathrm{I}_{\mathrm{SERS}}$ ) of pMA monolayer on SERS substrate to the intensities $\left(\mathrm{I}_{\mathrm{RS}}\right)$ of the corresponding unenhanced signals from neat pMA films of known thickness [18]. $N_{\text {surf }}$ and $N_{\text {vol }}$ correspond to the number of pMA molecules adsorbed on a SERS substrate surface and in a neat pMA film, respectively. The largest estimated $\mathrm{EF}$ of $2 \times 10^{6}$ was achieved for $\mathrm{Au}$ nanolayers (E). However, high EFs in the range of $10^{5}-10^{6}$ were also observed for layers (C), (D) and (F) (Fig. 2 and Fig. 4).

In conclusion, it was shown that semicontinuous $\mathrm{Au}$ layers with increased extinction in the Vis-NIR range can be fabricated by using a simple and reproducible method. The fabricated nanostructures exhibit strong SERS signal enhancement for simple analytes. Studies are underway to test their applicability for SERS measurements of more complex biological materials.

The authors gratefully acknowledge the support of this work by the National Centre for Research and Development (NCBiR) through Grant O N507 282540. The assistance of Dr. D. Zasada in the SEM measurements is also gratefully acknowledged.

\section{References}

[1] B. Sharma, R.R. Frontiera, A-I. Henry, E. Ringe, R.P. Van Duyne, Mater. Today 15, 16 (2012).

[2] G.C. Schatz, M.A. Young, R.P. Van Duyne, In Topics in Applied Physics 103, 19-46: Surface Enhanced Raman Scattering Physics and Applications; Kneipp, K., Moskovits M., Kneipp, H. Eds.; (Springer, New York, 2006).

[3] T. Vo-Dinh, T.A.C.-Trend. Anal. Chem. 17, 557 (1998).

[4] G.A. Baker, D.S. Moore, Anal. Bioanal. Chem. 382, 1751 (2005).

[5] M. Fan, G.F.S. Andrade, A.G. Brolo, Anal. Chim. Acta 693, 7, (2011).

[6] K. Kneipp, Y. Wang, H. Kneipp, L.T. Perelman, I. Itzkan, R. Dasari, M.S. Feld, Phys. Rev. Lett. 78, 1667 (1997).

[7] J.P. Camden, J.A. Dieringer, J. Zhao, R.P. Van Duyne, Acc. Chem. Res. 41, 1653 (2008).

[8] V.P. Drachev, M.D. Thoreson, V. Nashine, E.N. Khaliullin, D. Ben-Amotz, V.J. Davisson, V.M. Shalaev. J. Raman, Spectrosc. 36, 648 (2005).

[9] D. J. Semin, K. L. Rowlen Anal. Chem. 66, 4324 (1999).

[10] J.B. Jackson, N.J. Halas. Proc. Natl. Acad. Sci. USA 101, 17930 (2004).

[11] H. Wang, C.S. Levin, N.J. Halas J. Am. Chem. Soc. 127, 14992 (2005).

[12] K.R. Brown, L.A. Lyon, A.P. Fox, B.D. Reiss, M.J. Natan, Chem. Mater. 12, 314 (2000).

[13] K.C. Grabar, R.G. Freeman, M.B. Hommer, M.J. Natan, Anal. Chem. 67, 735 (1995).

[14] K. Fujiwara, H. Kasaya, N. Ogawa, Anal. Sci. 25, 241 (2009).

[15] J. Kimling, M. Maier, B. Okenve, V. Kotaidis, H. Ballot, A. Plech, J. Phys. Chem. B 110, 15700 (2006).

[16] K.C. Grabar, K.J. Allison, B.E. Baker, R.M. Bright, K.R. Brown, R.G. Freeman, A.P. Fox, C.D. Keating, M.D. Musick, M.J. Natan, Langmuir 12, 2353 (1996).

[17] J.H. Moon, J.H. Kim, K.-J. Kim, T.-H. Kang, B. Kim, Ch.H. Kim, J.H. Hahn, J.W. Park, Langmuir 13, 4305 (1997).

[18] B. Yan, A. Thubagere, W.R. Premasiri, L.D. Ziegler, L. Dal Negro, B.M. Reinhard, ACS Nano 3, 1190 (2009).

[19] N. Mohri, S. Matsushita, M. Inoue, K. Yoshikawa, Langmuir 14, 2343 (1998). 